\title{
Dark Matter or Additional Gravitational Forces Generated by Non-Spherical Mass Distributions?
}

\author{
Enbang Li \\ School of Physics, Faculty of Engineering and Information Sciences \\ University of Wollongong, NSW 2522, Australia \\ enbang@uow.edu.au
}

Received 23 November 2016

Revised 24 December 2016

Accepted 5 February 2017

Published 2 March 2017

\begin{abstract}
The measured orbital velocity distributions of stars in galaxies and the observed gravitational lensing effects in galaxy clusters suggest that there should be more mass than that can be explained by the visible mass of stars, gas and dust in the galaxies. This unseen mass or matter, generally referred to as dark matter, has puzzled physicists for a few decades and has now become one of the greatest unsolved mysteries in modern science. So far, all of the efforts aiming to generate and detect the exotic dark matter substance have yielded negative results. Here, starting from Newton's law of gravity, we show that the spherical mass distribution models originally employed for estimating the masses of galaxies could cause the discrepancy between the actual masses and those calculated from the rotational velocities. It is demonstrated that additional gravitational effects are generated from non-spherical mass distributions in the cosmic structures. The currently observed rotation curves and gravitational lensing effects in galaxies and galaxy clusters could be explained under the frameworks of Newtonian gravity and Einstein's general theory of relativity when proper mass distributions are considered.
\end{abstract}

Keywords: Dark matter; gravitational force; non-spherical mass distribution; Newton gravity; Einstein's general theory of relativity.

\section{Introduction}

According to Newton's law of gravity, for a particle of mass $m$ orbiting with velocity $v$ around a spherical distribution of mass $M$, at distance $r$ from the center, the centrifugal equilibrium condition requires $m v^{2} / r=G m M / r^{2}$, where $G$ is the Newton's constant of gravitation. If we express the velocity as a function of $r$, then a rotation curve with a Keplerian declining form $v(r)=(G M / r)^{1 / 2}$ would be expected. For some galaxies, like the Milky Way and the Andromeda (M31), however, instead of this Keplerian declining behavior, concrete measurements of the stars in the

This is an Open Access article published by World Scientific Publishing Company. It is distributed under the terms of the Creative Commons Attribution 4.0 (CC-BY) License. Further distribution of this work is permitted, provided the original work is properly cited. 
galaxy disks have shown that the rotation velocity distributions are almost constant throughout much of the galaxy disks. ${ }^{1,2}$ More and more optical and radio observations indicate that all spiral galaxies that so far have been studied commonly possess flat rotation curves. ${ }^{3,4}$ This fact leads to a remarkable conclusion: there are missing masses from the galaxies. On the other hand, the observed gravitational lensing effects which are caused by the light deflections predicted by Einstein's general theory of relativity also indicate that there must be more masses in the lensing galaxies to account for the observed phenomena. ${ }^{5-8}$

In order to explain the flat rotational curves, dark matter was first hypothesized. ${ }^{9}$ By its definition, different from the ordinary matter which is made of baryonic particles, dark matter is non-luminous and only interacts with ordinary matter through gravitation. It is now believed that the universe contains more dark matter than ordinary matter, and dark matter plays a pivotal role in the formation of cosmic structure of the universe. ${ }^{10-12}$ Dark matter has puzzled physicists for a few decades and has now become one of the greatest unsolved mysteries in modern science. ${ }^{13,14}$ So far, various candidates including massive compact halo objects (MACHOs), heavy neutrinos, and dark matter particles such as weakly interacting massive particles (WIMPs) which are predicted by various extension of the standard model of particle physics, have been proposed. ${ }^{5,15}$ Detection or generation of dark matter particles has now become an extremely active research area in particle physics. ${ }^{16,17}$ So far, however, none of the experiments aiming to generate and detect dark matter particles have been successful. ${ }^{18}$

As an alternative to dark matter, modified Newtonian dynamics (MOND) theory has also been proposed. ${ }^{19}$ MOND can successfully explain the flat rotation curves of spiral galaxies, but it is difficult to satisfy the well-established tests of Einstein's general theory of relativity, including gravitational lensing. ${ }^{20}$

Einstein's general theory of relativity has passed various tests and the recent successful detection of gravitational waves has added one more evidence for demonstrating the correctness and power of the theory. ${ }^{21}$ Under current circumstance, while more efforts are currently being made for improving the detection sensitivity or extending the range of searching, ${ }^{22,23}$ it is necessary to re-examine every factor in dealing with the gravitation forces in large-scale structures such as galaxies and galaxy clusters.

\section{Results}

In case of a star orbiting around the center of a galaxy, the combined force exerted on the star is the vector sum of all gravitational forces by every object (including stars, gas, and dust) contained in the galaxy. According to Newton's third theorem on gravitation, a mass that is inside a homoeoid experiences no net gravitational force from the homoeoid. ${ }^{24}$ This means that for an ellipsoidal mass distribution, only mass inside the ellipsoid needs to be considered in calculating gravitational force. As a special case, a spherical mass distribution satisfies the condition required by the theorem, and also can be further treated as a point source (with its total mass) 
located in its center for both near-field and far-field situations. However, for nonspherical mass distributions, this statement holds only for the far-field and could become invalid for the near-field.

Galaxies all have very different shapes, from ellipticals, spirals, barred spirals, to irregular galaxies, and their mass distributions, in most cases, are complex and significantly different from spherical. It should be noted that spherical models were employed by both Zwicky and Rubin in their early studies when dark matter was postulated.

The effects of non-spherical mass distributions of galaxies have been considered in various publications (see, for example ${ }^{2,24,25}$ ). The aim of the study is to demonstrate that additional gravitational forces could be generated when the mass distributions are non-spherical by using simplified models.

The near-field gravitational forces generated by non-spherical mass distributions for various shapes can be calculated (see Methods) and the results show that the near-field gravitational force distributions strongly depend on the mass distributions and can significantly deviate from those generated by spherical mass-distributions.

Shown in Fig. 1 are the calculated gravitational force distributions for two different mass distributions: an oblate spheroid and a disk. For comparison purposes, the calculated gravitational forces are normalized by that generated from a simplified point mass which is equivalent to a spherical mass distribution. Plotted in Fig. 1 are values of a factor $k_{g}$ defined as $k_{g}=F / F_{0}$, where $F$ is the gravitational force between the test mass $m$ and the distributed mass with a total mass of $M$; and $F_{0}$ is the gravitational force between the test mass $m$ and the simplified point mass $M$. Therefore, for a non-spherical mass distribution, $k_{g}$ represents how much the gravitational force deviates from that generated by a spherical mass-distribution.

It can be easily verified that for a spherical mass distribution, $k_{g}$ is constantly unity in the space out of the distributed mass, both in near- and far-field conditions.

For the oblate spheroid or revolving ellipsoid (the length of semi-minor axis is half of that of semi-major axis, $R$ ) mass distribution considered here, it is clear that near the ellipsoid, $k_{g}$ increases to values larger than 1 along the semi-major axis direction, indicating increased gravitational forces. Along the semi-minor axis direction, we see that $k_{g}<1$.

The variations of gravitational forces will become more obvious with the increase of the oblateness or flatness of the mass distribution. The extreme case is a disk-like mass distribution, as shown in Figs. 1(c) and 1(d). In the vicinity of the disk edge, $k_{g}$ can reach values which are much larger than 1 , depending on the distance from the disk edge. For a case where the distance is $0.01 \%$ of the disk radius $(R), k_{g}$ reaches 6 . This means that a particle locating at that point will experience a gravitational force which is more than six times stronger than that generated by a point source with the same mass locating at the center of the disk. In other words, a disk of mass $M$ acting on a particle at the disk edge is equivalent to a spherically distributed mass of $6 \mathrm{M}$. More detailed calculations show that when the distance between the particle and the disk edge is further reduced, $k_{g}$ will increase more rapidly. 


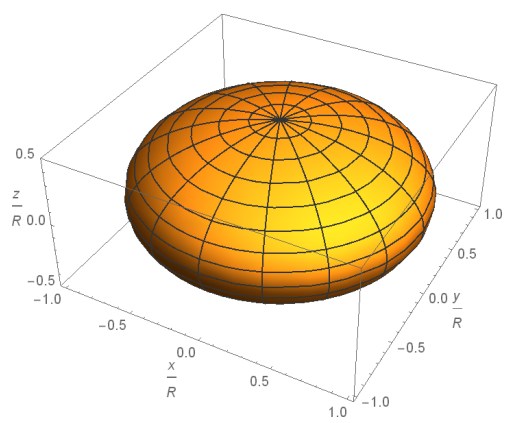

(a)

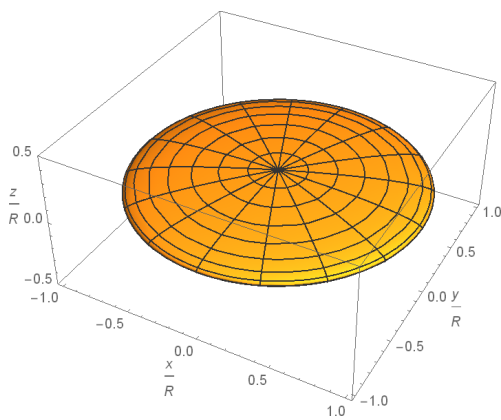

(c)

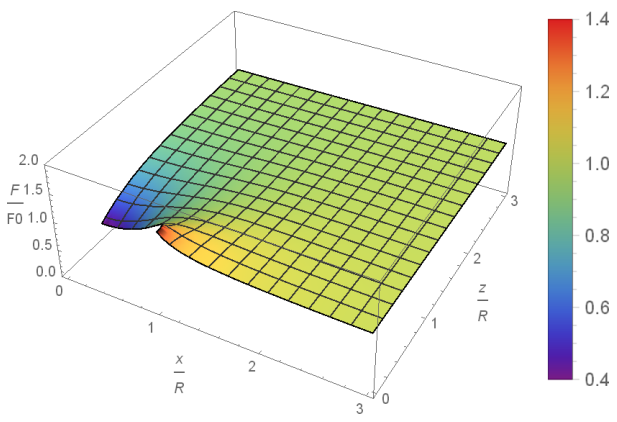

(b)

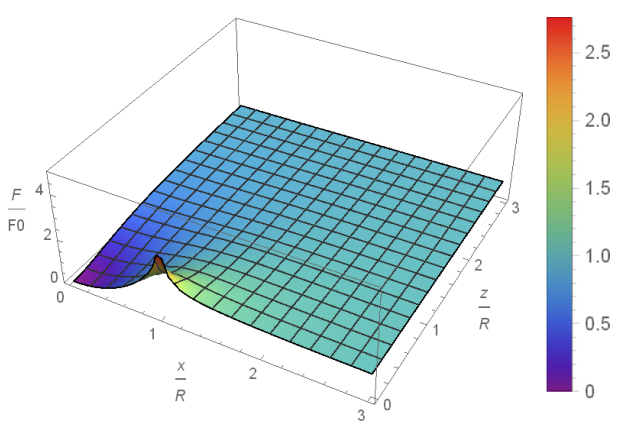

(d)

Fig. 1. Calculated gravitational forces for two different mass distributions. (a) Oblate spheroid (ellipsoid) mass distribution. The length of semi-minor axis is half of that of semi-major axis and within the ellipsoid a uniform mass density is assumed. (b) Gravitational force distribution generated by the ellipsoid massdistribution in the $\mathrm{x}-\mathrm{z}$ plane. The vertical axis represents $k_{g}=F / F_{0}$, where $F$ is the gravitational force between and the test mass $m$ and the distributed mass with a total mass of $M$; and $F_{0}$ is the gravitational force between the test mass $m$ and the simplified point mass $M . F / F_{0}$ represents how much the gravitational force deviates from that generated by a spherical mass-distribution. (c) Disk mass distribution. The surface mass density is constant across the disk. (d) Calculated gravitational force distribution generated by the disk. A significant enhancement in the gravitational force is obvious in the vicinity of the disk edge.

In the Milky Way galaxy which can be simplified as a disk, there are more than 100 billion stars. If we take the radius of the Milky Way as $R_{M}=25 \mathrm{kpc}$, then the average distance between two adjacent stars would be less than $10^{-5} R_{M}$. The distance between the Sun and its nearest star Proxima Centauri is about $1.3 \mathrm{pc}$ and the distance from the Sun to the galactic center is about $8.0 \mathrm{kpc}$, which gives a ratio of $1.6 \times 10^{-4}$.

The Sun's orbiting velocity was measured as $220 \mathrm{~km} / \mathrm{s}$. If one uses a spheroidal model for estimating the mass of our galaxy inside the Sun's orbit, that is, $M=r v^{2} / G$, then the result is $9.0 \times 10^{10} M_{\odot}$, which, in fact, is overestimated by a factor of $k_{g}$. 
According to Einstein's general theory of relativity, the gravitational attraction of a mass $M$ deflects light and generates gravitational lensing effect. ${ }^{26}$ The deflection angle for a light ray passing by the mass $M$ with a distance $R$ from the center is $\alpha=2 R_{s} / R=4 G M / R c^{2}$, where $R_{s}=2 G M / c^{2}$ is the Schwarzchild radius, $c$ is the speed of light. If the light ray passes through the disk edge in the disk plane, the enhanced gravitational potential will bend the light ray as a mass of $k_{g} M$ does, meaning that the deflection angle will be enhanced by $k_{g}$ times. Therefore, when the gravitational lensing effects are used to estimate the mass of the lensing galaxy or galaxy cluster, the mass would be exaggerated by a factor of $k_{g}$.

One can go further from the disk-like mass distribution to consider barred structures. For a bar of length of $L$ with a uniform mass distribution, an analytical expression of $k_{g}$ for the locations along the bar direction can be obtained as $k_{g}=L /(4 d)$, where $d$ is the distance from the bar end (see Methods). It can be seen that a significant gravitational force enhancement would be expected in the vicinity of the bar end. As an example, if $d / L=1 \%$, then $k_{g}=25$.

From the detailed calculations of the gravitational forces for different mass distributions, we can conclude that for non-spherical mass distributions, in the nearfield, there exist some "hot spots" where the gravitational forces are enhanced. The enhancement factor strongly depends on the shape of the mass distribution of interest. As shown in Fig. 1, for an ellipsoidal distribution, $k_{g}$ will increase with the eccentricity. Only spheroidal mass distributions can produce Keplerian type of rotation curves as predicted by $v(r)=(G M / r)^{1 / 2}$ and extra gravitational forces generated by the flattened spheroidal mass distributions will allow a star orbiting the distributed mass with a velocity faster than that predicted by the Kepler law. It should be pointed out that away from the distributed mass by two or three times the radius $(R), k_{g}$ is almost constant and approaching unity, indicating that the distributed mass can be treated as a point mass for the far-field.

Shown in Fig. 2 is the observed rotation curve for the Milky Way along its radius. As can be seen there is a small region $(R<0.8 \mathrm{kpc}$, labeled with $\mathrm{A})$ where the rotation velocity is declining with a Keplerian fashion. Since there is a globular nucleus in the center of the galaxy, it could be an indication that the mass distribution is close to spheroidal. The deviation from the Keplerian curve beyond $0.8 \mathrm{kpc}$ suggests that the mass distribution starts to become non-spherical. The two low rotation velocity areas labeled by $\mathrm{B}$ and $\mathrm{C}$ respectively could be corresponding to the low number density regions between the central bar and the Scutum-Centaurus arm, and between the Sun and the Perseus arm of the Galaxy, since the stars in these areas have larger distances from the disk inside and consequently smaller gravitational force enhancement factors.

In previous studies, the Milky Way galaxy has been modeled as a thin or thick disk (non-spherical) with an exponential function. ${ }^{27}$ However, the mass of the baryonic matter of the galaxy cannot explain all details of rotational curves and dark matter halos are still needed to make the rotation curves flat. ${ }^{2}$ The reason could be due to the exponential mass distribution used in the previous studies. With a new 


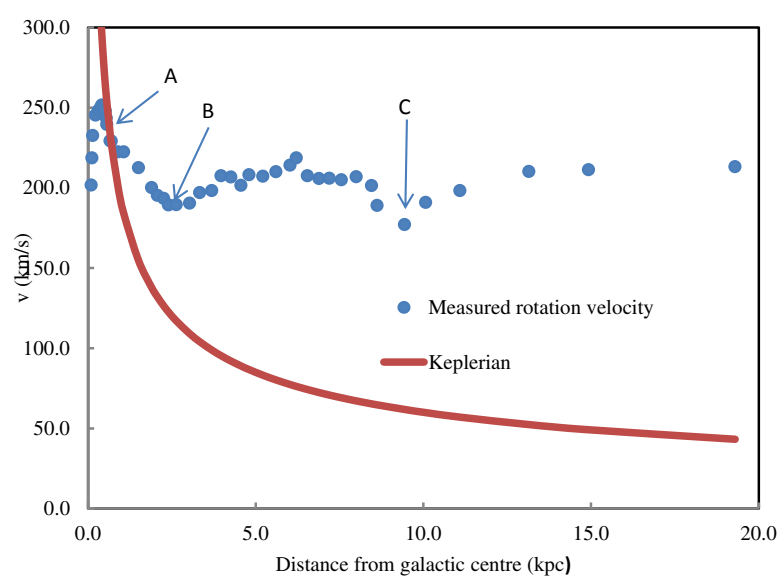

Fig. 2. Measured rotation velocities of stars in the Milky Way galaxy as a function of the distance from the galactic center. The data were obtained from the trend line of the observed rotation velocities presented in Fig. 4 of Ref. 4. The red curve is a Keplerian type fit to the declining rotation velocities within a distance of $0.8 \mathrm{kpc}$ (labeled by A), indicating that the mass-distribution in the galactic center of the Milky Way could be represented by a spheroid in the region of radius of about $0.8 \mathrm{kpc}$. Using the Keplerian curve fitting parameters, the mass within $0.8 \mathrm{kpc}$ radius is estimated as $5.6 \times 10^{6} M_{\odot}$. The deviation from the Keplerian curve beyond $0.8 \mathrm{kpc}$ indicates that the mass-distribution starts to become non-spherical. The two low rotation velocity areas labeled by $\mathrm{B}$ and $\mathrm{C}$ respectively could be corresponding to the low number density regions between the central bar and the Scutum-Centaurus arm, and between the Sun and the Perseus arm of the Galaxy.

mass distribution model by using the recently released Gaia billion-star map, a flat rotation curve which reproduces the key observed features of the Milky Way with no need for a dark halo has been obtained. The details on this topic are out of the scope of this paper, and will be discussed in a forthcoming publication. ${ }^{28}$

\section{Discussion}

Newton's theorems on gravitation guarantee that the gravitational force between a test mass and a spherical mass-distribution is constantly equal to that between the test mass and an equivalent point mass in both near-field and far-field. ${ }^{24}$ Extra care should be taken when applying this equivalence principle to large-scale structures with complex mass distributions, like galaxies and galaxy clusters. The gravitational force calculations presented in this work clearly show that for non-spherical mass distributions, significant differences in gravitation effects could be produced in the near-field. It is the near-field gravitational effects that play critical roles in determining the overall kinematic characteristics such as the rotation curves in galaxies, and in producing gravitational lensing effects. As can be appreciated, for nonspherical galaxies or galaxy clusters, if the spheroidal model is employed in galactic dynamics to estimate the masses within galaxies, then the results could be overestimated and consequently large discrepancies between the actual masses and those calculated from the rotational velocities might be produced. The additional 
gravitational forces generated from non-spherical mass distributions in the cosmic structures could be responsible for the currently observed flat rotation curves and gravitational lensing effects in galaxies and galaxy clusters. All of the observed phenomena could be explained under the frameworks of Newtonian gravity and Einstein's general theory of relativity when proper mass distributions are considered. Could the so-called dark matter be the additional gravitational effects generated by the non-spherical mass-distributions of the cosmic structures?

\section{Methods}

We consider a distributed object of total mass $M$ with rotational symmetry located in a coordinate system $(x, y, z)$, with the center of mass of the object being at the origin of the coordinate system and the symmetry axis being along the $z$ axis. The $x$ - and $z$-components of the gravitational force between a particle (test mass) of mass $m$ located at $\left(x_{0}, 0, z_{0}\right)$ and the distributed mass can be expressed as

$$
\begin{aligned}
& F_{x}=G m \int \frac{\left(x-x_{0}\right) \sigma}{\left[\left(x-x_{0}\right)^{2}+y^{2}+\left(z-z_{0}\right)^{2}\right]^{3 / 2}} d V, \\
& F_{z}=G m \int \frac{\left(z-z_{0}\right) \sigma}{\left[\left(x-x_{0}\right)^{2}+y^{2}+\left(z-z_{0}\right)^{2}\right]^{3 / 2}} d V,
\end{aligned}
$$

where $G$ is the Newton's constant of gravitation; $\sigma$ is the mass density. The integrations are performed over the volume of the distributed mass. It is obvious that the $y$-components of the gravitational force vanishes because of the symmetry. Here, we assume that the particle is located outside the distributed mass.

Then, the total gravitational force between $m$ and $M$ can be written as

$$
F=\left(F_{x}^{2}+F_{z}^{2}\right)^{1 / 2} .
$$

If we replace the distributed mass by a point source, the gravitational force can be expressed as

$$
F_{0}=G m M /\left(x_{0}^{2}+z_{0}^{2}\right) .
$$

We introduce a factor $k_{g}$ as $k_{g}=F / F_{0}$ to describe the deviation of the gravitation force from that of the point source equivalence. It can be verified that for a spherical mass distribution, $k_{g}$ is constantly unity for any particle locations outside the distributed mass. For non-spherical mass distributions, $k_{g}$ becomes position-dependent and can take values larger or smaller than 1 . Once the mass distribution is given, $k_{g}$ can be calculated for any point with coordinates $\left(x_{0}, 0, z_{0}\right)$.

For a two-dimensional mass distribution, the integrations should be performed over a surface with $\sigma$ representing a surface density.

With a special case, where a straight bar of a line density and a particle, both positioning along the $x$-axis, one can complete the integration and obtain

$$
F=F_{x}=G m M /\left(x_{0}^{2}-L^{2} / 4\right),
$$


where $L$ is the length of the bar. When the particle is close to the bar end, $k_{g}=F / F_{0} \cong L / 4 d, d$ is the distance from the particle to bar end, and $d \ll L$.

\section{Conflict of Interest}

The author declares no competing financial interests.

\section{References}

1. V. C. Rubin, The rotation of spiral galaxies, Science $\mathbf{2 2 0}$ (1983), 1339-1344, doi: 10.1126/ science.220.4604.1339.

2. F. Iocco, M. Pato and G. Bertone, Evidence for dark matter in the inner Milky Way, Nat. Phys. 11 (2015) 245-248, doi: 10.1038/nphys3237.

3. Y. Sofue and V. Rubin, Rotation curves of spiral galaxies, Ann. Rev. Astron. Astrophys. 39 (2001) 137-174, doi: 10.1146/annurev.astro.39.1.137.

4. Y. Sofue, Pseudo rotation curve connecting the galaxy, dark halo, and local group, Publ. Astron. Soc. Jpn. 61 (2009) 153-161, doi: 10.1093/pasj/61.2.153.

5. C. Alcock et al., Possible gravitational microlensing of a star in the large magellanic cloud, Nature 365 (1993) 621-623, doi: 10.1038/365621a0.

6. E. Aubourg et al., Evidence for gravitational microlensing by dark objects in the galactic halo, Nature 365 (1993) 623-625, doi: 10.1038/365623a0.

7. D. Clowe, A. Gonzalez and M. Markevitch, Weak-lensing mass reconstruction of the interacting cluster 1E 0657-558: Direct evidence for the existence of dark matter, Astrophys. J. 604 (2004) 596-603, doi: 10.1086/381970.

8. D. Clowe et al., A direct empirical proof of the existence of dark matter, Astrophys. J. 648 (2006) L109-L113, doi: 10.1086/508162.

9. F. Zwicky, Spectral displacement of extra-galactic nebulae,Helv. Phys. Acta 6 (1933) $110-127$.

10. V. C. Rubin, Stars, galaxies, cosmos - the past decade, the next decade, Science 209 (1980) 64-71, doi: 10.1126/science.209.4452.64.

11. D. N. Spergel, The dark side of cosmology: Dark matter and dark energy, Science $\mathbf{3 4 7}$ (2015) 1100-1102, doi: 10.1126/science.aaa0980.

12. R. D. Blandford, A century of general relativity: Astrophysics and cosmology, Science 347 (2015) 1103-1108, doi: 10.1126/science.aaa4033.

13. M. Davis, G. Efstathiou, C. S. Frenk and S. D. M. White, The end of cold dark matter? Nature 356 (1992) 489-494, doi: 10.1038/356489a0.

14. J. P. Ostriker and P. Steinhardt, New light on dark matter, Science 300 (2003) 19091913, doi: 10.1126/science.1085976.

15. J. L. Feng, Dark matter candidates from particle physics and methods of detection, Annu. Rev. Astron. Astrophys. 48 (2010) 495-545, doi: 10.1146/annurev-astro-082708-101659.

16. G. Bertone, The moment of truth for WIMP dark matter, Nature 468 (2010) 389-393, doi: 10.1038/nature09509.

17. A. Pontzen and F. Governato, Cold dark matter heats up, Nature 506 (2014) 171-178, doi: $10.1038 /$ nature 12953 .

18. D. S. Akerib et al., First Results from the LUX dark matter experiment at the sanford underground research facility, Phys. Rev. Lett. 112 (2014), doi: 10.1103/PhysRevLett.112.091303.

19. M. Milgrom, A modification of the newtonian dynamics as a possible alternative to the hidden mass hypothesis, Astrophys. J. 270 (1983) 365-370, doi: 10.1086/161130. 
20. J. D. Bekenstein, The modified Newtonian dynamics - MOND and its implications for new physics, Contemp. Phys. 47 (2006) 387-403, doi: 10.1080/00107510701244055.

21. B. P. Abbott et al., Observation of gravitational waves from a binary black hole merger, Phys. Rev. Lett. 116 (2016), doi: 10.1103/PhysRevLett.116.061102.

22. M. Livio and J. Silk, Broaden the search for dark matter, Nature 507 (2014) 29-31.

23. E. Gibney, C. Biever and D. Castelvecchi, Dark-matter probe launches era of Chinese space science, Nature $\mathbf{5 2 8}$ (2015) 443-444.

24. J. Binney and S. Tremaine, Galactic Dynamics (Princeton University Press, 1987).

25. J. T. A. de Jong et al., Mapping the stellar structure of the milky way thick disk and halo using SEGUE photometry, Astrophys. J. 714 (2010) 663-674.

26. J. B. Hartle, Gravity : An Introduction to Einstein's General Relativity (Addison-Wesley, 2003).

27. Y. Sofue, Grand rotation curve and dark-matter halo in the milky way galaxy, Publ. Astron. Soc. Jpn 64 (2012), doi: 10.1093/pasj/64.4.75 (2012).

28. E. Li, Modelling mass distribution of the Milky Way galaxy using Gaia's billion-star map, arXiv:1612.07781. 